\title{
Enhanced ionization of hydrogen molecular ions in an intense laser field via a multiphoton resonance
}

\author{
Ying-Jun Jin, ${ }^{1}$ Xiao-Min Tong, ${ }^{1,2, *}$ and Nobuyuki Toshima ${ }^{1}$ \\ ${ }^{1}$ Institute of Materials Science, Graduate School of Pure and Applied Sciences, University of Tsukuba, \\ 1-1-1 Tennodai, Tsukuba, Ibaraki 305-8573, Japan \\ ${ }^{2}$ Center for Computational Sciences, University of Tsukuba, 1-1-1 Tennodai, Tsukuba, Ibaraki 305-8577, Japan
}

(Received 17 September 2009; published 15 January 2010)

\begin{abstract}
Multiphoton ionization of hydrogen molecular ions in a 480-nm intense laser field is investigated by solving the time-dependent Schrödinger equation numerically in prolate spheroidal coordinates. We discretize space on a generalized pseudospectral grid and propagate the electronic wave function using a second-order split-operator method. By including and excluding the $2 p \sigma_{u}$ state in the basis expansion, we confirm that the observed $10-\mathrm{eV}$ peak in a recent experiment [Litvinyuk et al., New J. Phys. 10, 083011 (2008)] comes from the enhanced ionization via three-photon resonant excitation of the molecular ions. By folding the calculated ionization rates with the vibrational density distribution, the kinetic energy release spectra are obtained, which are in reasonable agreement with the experimental measurement. Furthermore, using this enhanced ionization, a pump-probe experiment is suggested to trace the vibrational wave packet.
\end{abstract}

DOI: 10.1103/PhysRevA.81.013408

PACS number(s): $33.80 . \mathrm{Rv}, 42.50 . \mathrm{Hz}, 34.50 . \mathrm{Gb}$

\section{INTRODUCTION}

Double ionization of hydrogen molecules in an intense laser field has been studied extensively both experimentally and theoretically. The double ionization is classified into nonsequential (rescattering-induced) double ionization and sequential double ionization. Niikura et al. [1] first observed several high-energy peaks in the kinetic energy release (KER) spectra, and the peaks were attributed to the rescatteringelectron-induced dissociation of hydrogen molecular ions. Alnaser et al. [2] measured the two $\mathrm{D}^{+}$ions coincidentally after dissociation of the $\mathrm{D}_{2}$ molecule and found that the peaks come from the rescattering-electron-induced ionization, a finding consistent with the theoretical study [3]. A later experiment [4] even shaped the laser pulse to select a specified return of the rescattering electron. The nonsequential double ionization of hydrogen molecules is well explained by the rescattering model [5], and the time interval between the first and second ionizations can be used to make a molecular clock $[1,6]$. Other low-energy peaks occur in the double ionization of hydrogen molecules. These peaks were predicted by Zuo and Bandrauk [7] as charge-resonance-enhanced ionization (CREI) and were observed in the experiment [8]. We can classify this process as sequential double ionization since it does not associate with the rescattering process. CREI plays an important role for a relatively large nuclear distance when the laser intensity is not too high. If the laser intensity is further increased, the molecules are singly ionized at first before the laser reaches its peak strength, and the residual molecular ions are ionized when the laser field is close to the peak. In this two-step ionization, the time interval between the first and second ionizations can be used to measure time in a femtosecond regime $[9,10]$. The mechanisms of double ionization of hydrogen molecules seem to be well known.

Recently, Litvinyuk et al. [11] observed a high-energy peak in the KER spectra when they doubly ionized $\mathrm{D}_{2}$ molecules using a $480-\mathrm{nm}$ intense laser. The observed peak cannot be

\footnotetext{
*tong.xiaomin.ga@u.tsukuba.ac.jp
}

explained by the nonsequential double ionization or CREI since the peak position is sensitive not to the laser intensity but to the laser center wavelength. They speculated that the peak originates from the excitation by the three-photon resonance to the excited state $2 p \sigma_{u}$, and sequentially the excited state is ionized by the laser field.

This could be another type of sequential double ionization of hydrogen molecules in the intense laser field. For sequential double ionization, we can study the first and second ionizations separately. We depict the second ionization, namely the ionization of the molecular ion, as an independent process and convolute the second ionization rates with the vibrational wave packet created in the first ionization of the neutral molecule to study the double-ionization process.

To investigate the physical origin of the observed peak, we perform a theoretical study of the ionization of hydrogen molecular ions in the intense laser field by solving the timedependent Schrödinger equation numerically. Since this is a two-center problem, we adopt prolate spheroidal coordinates. We discretize space on a grid and propagate the time-dependent wave function numerically [12]. The numerical procedure is similar to the study by Chu and Chu [13]. In this study, we confirm that the observed $10-\mathrm{eV}$ peak is associated with the enhanced ionization via the three-photon resonant excitation, which was not observed before.

Since this enhanced ionization occurs via a multiphoton resonant state that has a sharp energy distribution, we can measure the period of this created wave packet if we use it as a monitor to observe the vibrational wave packet created in the first ionization. Based on this idea, a numerical experiment was designed to use a pump laser to ionize a hydrogen molecule and to trace the evolution of the created vibrational wave packet using a 480-nm probe laser. This procedure is similar to pump-probe experiments using two infrared lasers $[14,15]$ but we can calibrate the vibrational period in the time-domain more accurately. The theoretical method is desribed in Sec. II and results are presented in Sec. III, followed by a summary. In this article, hydrogen molecule means either $\mathrm{H}_{2}$ or $\mathrm{D}_{2}$ and we do not distinguish between them unless denoted specifically. 


\section{THEORETICAL METHOD}

The electronic motion of a hydrogen molecular ion in a laser field is described by the following time-dependent Schrödinger equation (where atomic units $\hbar=m=e=1$ are used unless stated otherwise):

$$
i \frac{\partial}{\partial t} \Psi(t)=\left[H_{0}+V_{\text {ext }}(t)\right] \Psi(t)
$$

where $H_{0}$ is the Hamiltonian of hydrogen molecular ions and $V_{\text {ext }}(t)$ is the interaction of the electron with the external laser field. Under the Born-Oppenheimer approximation, $H_{0}$ is written as

$$
H_{0}=-\frac{1}{2} \nabla^{2}-\frac{1}{r_{1}}-\frac{1}{r_{2}}+\frac{1}{R},
$$

where $r_{1}\left(r_{2}\right)$ is the distance between the electron and the first (second) nucleus and $R$ is the internuclear distance. The time propagation of Eq. (1) is carried out by the second-order split-operator method in the energy representation [12]. At first we calculate the eigenfunctions of $H_{0}$ of the following time-independent Schrödinger equation numerically:

$$
\left(-\frac{\nabla^{2}}{2}-\frac{1}{r_{1}}-\frac{1}{r_{2}}+\frac{1}{R}\right) \psi(\mathbf{r})=E(R) \psi(\mathbf{r}),
$$

where $E(R)$ is the adiabatic eigenenergy, $\psi(\mathbf{r})$ the eigenfunction, and $\mathbf{r}$ the electron coordinates. In order to depict the two-center system, naturally we choose the prolate spheroidal coordinates $(\xi, \eta, \varphi)$, defined as

$$
\begin{gathered}
\xi=\frac{r_{1}+r_{2}}{R} \quad 1 \leqslant \xi \leqslant \infty, \\
\eta=\frac{r_{1}-r_{2}}{R} \quad-1 \leqslant \eta \leqslant 1,
\end{gathered}
$$

and $\varphi$ is the azimuthal angle with respect to the molecular axis. In the prolate spheroidal coordinates, Eq. (3) is written as

$$
\left[-\frac{\nabla^{2}}{2}-\frac{1}{a(\xi+\eta)}-\frac{1}{a(\xi-\eta)}\right] \psi(\xi, \eta, \varphi)=E \psi(\xi, \eta, \varphi),
$$

with $a=R / 2$ and

$$
\begin{aligned}
\nabla^{2}= & \frac{1}{a^{2}\left(\xi^{2}-\eta^{2}\right)}\left[\frac{\partial}{\partial \xi}\left(\xi^{2}-1\right) \frac{\partial}{\partial \xi}+\frac{\partial}{\partial \eta}\left(1-\eta^{2}\right) \frac{\partial}{\partial \eta}\right. \\
& \left.+\frac{\xi^{2}-\eta^{2}}{\left(\xi^{2}-1\right)\left(1-\eta^{2}\right)} \frac{\partial^{2}}{\partial \varphi^{2}}\right] .
\end{aligned}
$$

Since the Coulomb potentials do not depend on $\varphi$, the wave function can be expressed as

$$
\psi(\xi, \eta, \varphi)=\Phi^{m}(\xi, \eta) \frac{e^{i m \varphi}}{\sqrt{2 \pi}}
$$

and the equation is reduced to a two-dimensional problem as

$$
\left[-\frac{\nabla^{2}}{2}-\frac{1}{a(\xi+\eta)}-\frac{1}{a(\xi-\eta)}\right] \Phi^{m}(\xi, \eta)=E \Phi^{m}(\xi, \eta)
$$

and $-\partial^{2} / \partial \varphi^{2}$ is replaced with $m^{2}$ in Eq. (7). We map $\xi$ and $\eta$ onto $[-1: 1]$ as

$$
\begin{gathered}
\xi=1+L \frac{1+x}{1-x} \\
\eta=y
\end{gathered}
$$

and discretize $x, y$ using a generalized pseudospectral grid $[16,17]$, where $L$ is a mapping parameter used to adjust the grid distribution. The basis functions of the generalized pseudospectral method are

$$
\begin{gathered}
f_{i}(x)=\frac{1}{\left(1-x_{i}^{2}\right) P_{N}^{\prime}\left(x_{i}\right)} \frac{\left(1-x^{2}\right) P_{N}(x)}{x-x_{i}}, \\
f_{j}(y)=\frac{1}{P_{M}^{\prime}\left(y_{i}\right)} \frac{P_{M}(y)}{y-y_{i}} .
\end{gathered}
$$

Here $\left\{x_{i}\right\}\left(\left\{y_{i}\right\}\right)$ are the roots of the $N$ th-order (Mth-order) Legendre polynomials. We use different basis functions for $x$ and $y$ because the boundary conditions are different for $\xi$ and $\eta$. Using the preceding basis functions, we expand the wave function $\Phi^{m}(\xi, \eta) \cong \sum_{i, j} C_{i, j} f_{i}(x) f_{j}(y)$ and discretize Eq. (9). Diagonalizing the Hamiltonian, we get the eigenenergies $\left\{\epsilon_{k}\right\}$ and the eigenfunctions $\left\{\Phi_{k}^{m}(\xi, \eta)\right\}$.

We assume that the laser field is polarized along the molecular axis so that the electron laser field interaction is expressed as

$$
V_{\text {ext }}(t)=E_{0} a \xi \eta e^{-2 \ln 2 t^{2} / \tau^{2}} \cos (\omega t),
$$

where $E_{0}$ is the laser field strength, $\tau$ is the full width at half maximum of the laser pulse duration, and $\omega$ is the laser center frequency. The initial electronic state is the ground state $1 s \sigma_{g}$ with $m=0$. Since $m$ is a good quantum number in the present configuration, we drop the quantum number $m$ in the following expressions. The time propagation of the wave function from time $t$ to time $t+\Delta t$ is performed as

$$
\begin{aligned}
\Psi(\xi, \eta, t+\Delta t) \simeq & e^{-i H_{0} \Delta t / 2} \times e^{-i V(t) \Delta t} \\
& \times e^{-i H_{0} \Delta t / 2} \Psi(\xi, \eta, t),
\end{aligned}
$$

with the initial condition $|\Psi(t=-\infty)\rangle=\left|1 s \sigma_{g}\right\rangle$. To avoid the unphysical reflection of the electronic wave function at the boundary, we add an optical potential to $V_{\text {ext }}(t)$, where $\xi_{c}$ the starting position of the absorber:

$$
V_{\mathrm{opt}}= \begin{cases}0, & \text { if } \xi<\xi_{c} \\ -i V_{0}\left(\frac{\xi-\xi_{c}}{\xi_{\max }-\xi_{c}}\right), & \text { if } \xi \geqslant \xi_{c} .\end{cases}
$$

Using the basis set $\left\{\Phi_{k}(\xi, \eta)\right\}$ of hydrogen molecular ions obtained from Eq. (9), we propagate the time-dependent wave function as

$$
\begin{aligned}
C_{k}(t+\Delta t)= & \sum_{k^{\prime}} e^{-i \epsilon_{k} \Delta t / 2}\left\langle\Phi_{k}\left|e^{-i V_{\mathrm{ext}} \Delta t}\right| \Phi_{k^{\prime}}\right\rangle \\
& \times e^{-i \epsilon_{k^{\prime}} \Delta t / 2} C_{k^{\prime}}(t),
\end{aligned}
$$

with $C_{k}(t)=\left\langle\Phi_{k} \mid \Psi(t)\right\rangle$. We define a time-dependent ionization probability as

$$
P(t)=1-\sum_{\epsilon_{k}<0}\left|C_{k}(t)\right|^{2}
$$

and obtain the final ionization probability as $t \rightarrow \infty$.

So far we have assumed that the nuclear separation is fixed. Now let us consider the nuclear vibrational motion. Since the nuclei are very heavy compared to the electron, we assume that the laser field does not directly affect nuclear vibration. Thus, the vibrational ground state $\chi_{g}(R)$ of the neutral hydrogen molecule is promoted to the vibrational states of the hydrogen 
molecular ion by the laser through the first ionization by a sudden approximation (the Franck-Condon principle). The time evolution of the vibrational wave packet created at time $t_{0}$ satisfies the following Schrödinger equation:

$$
i \frac{\partial}{\partial t} \chi(R, t)=\left[-\frac{1}{2 \mu} \frac{d^{2}}{d R^{2}}+V(R)\right] \chi(R, t),
$$

where the initial condition $\chi\left(R, t=t_{0}\right)=\chi_{g}(R)$, and $V(R)$ is the adiabatic potential of the ground state of the hydrogen molecular ion. The vibrational wave packet is propagated in the pseudospectral grid. The term $\left|\chi\left(R, t-t_{0}\right)\right|^{2}$ stands for the wave packet density distribution at time $t$ when the molecule is ionized at time $t_{0}$. Folding the vibrational wave packet density with the calculated ionization rates, we obtain the KER spectra to be compared with the experimental measurements. In the present study, it is assumed that the ionization of molecular ions comes from the ground electronic state and we did not take into account the contribution of the wave packet associated with the excited electronic states (such as the $2 p \sigma_{u}$ state), which plays an important role in the dissociation process [18,19].

\section{RESULTS AND DISCUSSION}

To check the accuracy of our numerical code, we first calculated the ionization rates of hydrogen molecular ions in the infrared pulsed laser and obtained good agreement with other calculations $[7,20]$. CREI peaks were also observed. In our simulation, we use a pulsed laser as described in Eq. (14), and the averaged ionization rate is defined as

$$
R_{\text {avg }}=P(t=\infty) / \tau \text {. }
$$

Figure 1 shows the time-dependent ionization probabilities of hydrogen molecular ions at a fixed internuclear distance ( $R=2.4$ a.u.) for a 10 -fs pulsed laser with 480 -nm wavelength. We have used 280 points in $\xi, 15$ points in $\eta$, and 500 steps per optical cycle for time propagation. After diagonalizing the Hamiltonian without the laser field, we obtain about 800 bound electronic states. It is visible from the

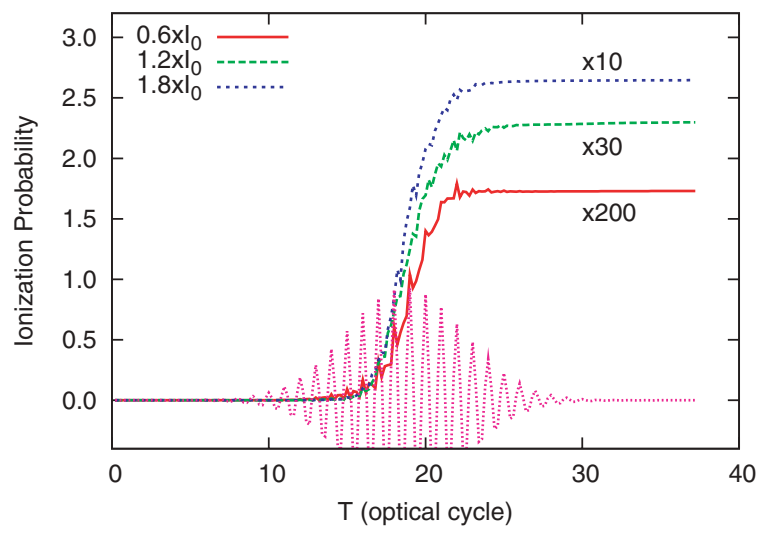

FIG. 1. (Color online) Time-dependent ionization probabilities of hydrogen molecular ions at a fixed internuclear distance $(R=$ 2.4 a.u.) for various laser intensities $\left(I_{0}=10^{14} \mathrm{~W} / \mathrm{cm}^{2}\right)$ with $480-\mathrm{nm}$ wavelength and 10-fs pulse duration. The pulsed laser field is also plotted in the figure. figure that the ionization happens when the laser field reaches its peak strength, and the ionization probabilities approach constants after the field vanishes. This figure shows that the propagation time is long enough to obtain the ionization rates. A longer pulse was also used to calculate the average ionization rate and the results agree with the use of a 10-fs pulsed laser. Some spikes appear in the time-dependent ionization probabilities after the laser field is turned on. These spikes come from the definition of the time-dependent ionization probability in Eq. (18). When the laser field is on, the field-free states are no longer the eigenstates of the system and the electron can hop between the continuum and the bound states in the field.

Figure 2 shows our simulated KER spectra and the experimental measurements [11]. The KER spectrum is calculated as

$$
\frac{d P}{d E}=\int_{-\infty}^{\infty} \int_{t_{0}}^{\infty} \chi^{2}\left(R, t-t_{0}\right) R_{\mathrm{avg}}(R) W\left(t_{0}\right)\left|\frac{d R}{d E}\right| d t d t_{0},
$$

where $E=1 / R$ and $W\left(t_{0}\right)$ is the molecular tunneling ionization rate [21] for the first ionization at time $t_{0}$. The experiment was done with a 100 -fs pulse for $\mathrm{D}_{2}$ molecules, so we use the same laser-pulse duration in the simulation. The calculated results are in agreement with the measurements, and peaks occur around $10 \mathrm{eV}$. There are some discrepancies
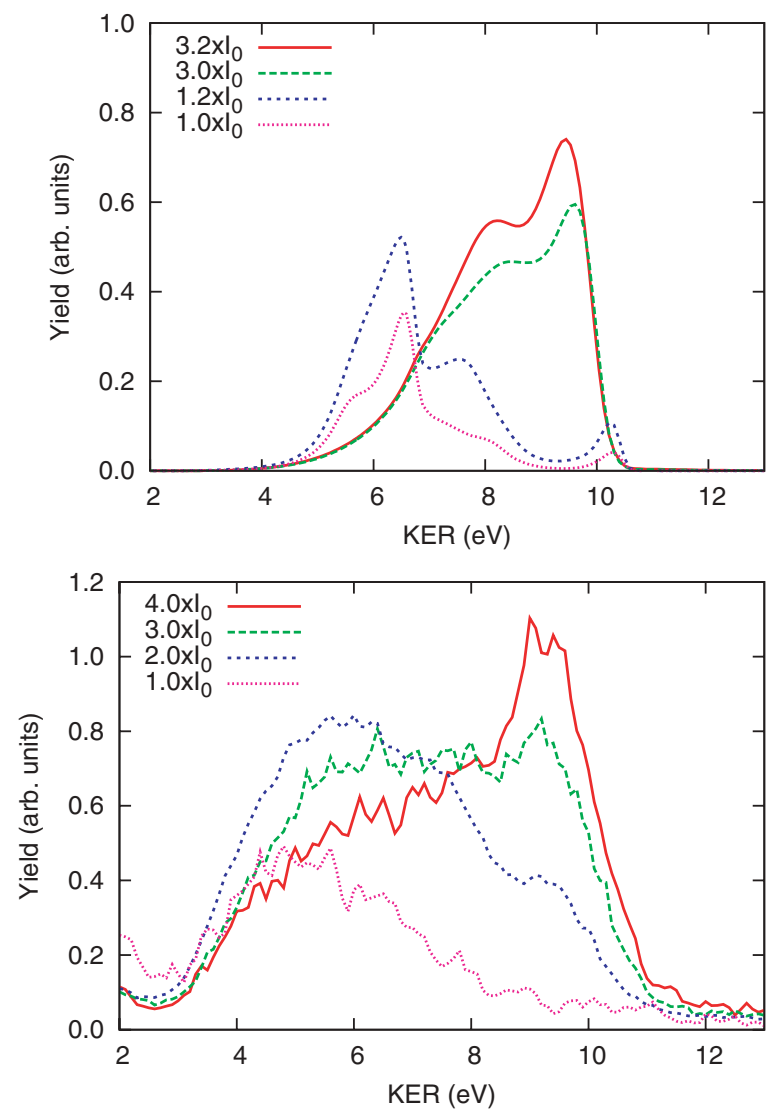

FIG. 2. (Color online) Simulated KER spectra (upper panel) due to the double ionization of $\mathrm{D}_{2}$ in $480-\mathrm{nm}$ pulsed laser fields. The experimental data are plotted in the lower panel for comparison. 


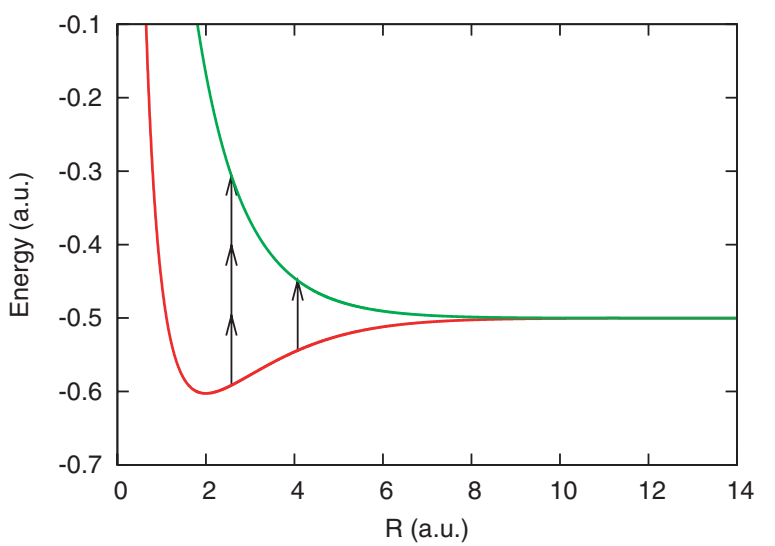

FIG. 3. (Color online) Adiabatic potential curves of hydrogen molecular ions. The length of the arrows indicates the energy of a single photon.

in detail between the theory and the experiment, but the overall structures are similar. In the current simulation, the laser field is assumed to be polarized along the molecular axis and the volume effect of the laser beam $[21,22]$ has not been taken into account. In our results, we see that the KER spectra are very sensitive to the laser intensity. Thus, these factors could be the origin of the discrepancies. We have not included the rescattering process, so the peaks are irrelevant. The main peak energy in Fig. 2 is about $10 \mathrm{eV}$, higher than the KER energy of CREI. Litvinyuk et al. [11] made a speculation that the peaks come from the ionization via a three-photon resonant excitation-a new double-ionization route that was not observed before. Their speculation was based on the fact that the three-photon energy is in coincidence with the transition energy between the $1 s \sigma_{g}$ and $2 p \sigma_{u}$, as shown in Fig. 3 at $R$, which corresponds to the KER peak position near $10 \mathrm{eV}$.

Figure 4 shows the ionization rates as a function of the internuclear distance for a given laser intensity. If we remove the contribution of $2 p \sigma_{u}$ artificially from the simulation, the peaks in the small- $R$ region disappear completely. The

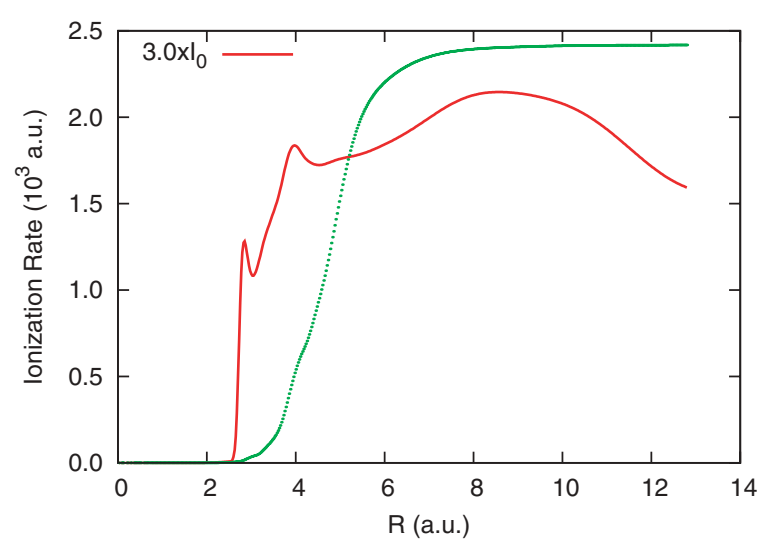

FIG. 4. (Color online) Ionization rates of hydrogen molecular ions in a 480-nm pulsed-laser field as a function of the internuclear distance. In the simulation, the $2 p \sigma_{u}$ state is included (solid line) and excluded (dotted line). energy difference between the $1 s \sigma_{g}$ and $2 p \sigma_{u}$ states at the peak position ( $R=2.8$ a.u.) coincides with the three-photon energy. The peak around $R=4.0$ is one-photon resonance and the broad peak around $R=9.0$ corresponds to CREI. In order to check whether the coincidence is accidental, the wavelength was varied from 400 to $600 \mathrm{~nm}$ and it was found that the position also moves to the place where the three-photon resonance occurs. In this way, we confirm that the new observed peaks are due to the ionization via the three-photon resonant excitation from $1 s \sigma_{g}$ to $2 p \sigma_{u}$. This process was not observed in previous experiments using the infrared laser because (1) the peak moves to a low-energy region where CREI becomes important and (2) the rescattering-induced double ionization also plays an important role there. The $2 p \sigma_{u}$ state serves two roles in the molecular-ion ionization. One is the role of an intermediate state through which the ground state is ionized. The other is the contribution to the perturbation of the ground state, which suppresses the ionization potential of the ground state due to the laser-molecular ion interactions. The first one is important at a small $R$, where the energies of $1 s \sigma_{g}$ and $2 p \sigma_{u}$ are well separated and the ionization rate is increased. The second one is important at a large $R$, where, to the contrary, the $1 s \sigma_{g}$ and $2 p \sigma_{u}$ states are almost degenerated and the ionization rate is suppressed. This may explain why the averaged ionization rates decrease at a small $R$ and increase at a large $R$ when we remove the $2 p \sigma_{u}$ state.

To see the contributions of the $2 p \sigma_{u}$ state, we plot the timedependent populations of the $1 s \sigma_{g}$ (solid line), $2 p \sigma_{u}$ (dashed line), and the sum of all the other electronic excited states (dotted line) in Fig. 5 at four different nuclear separations, which correspond respectively to the three-photon resonant peak [Fig. 5(a)], the minimum between the three- and onephoton resonant peaks [Fig. 5(b)], the one-photon resonant peak [Fig. 5(c)], and the CREI peak [Fig. 5(d)] from Fig. 4. Overall, we see that the populations of the $2 p \sigma_{u}$ state are larger than that of the sum of all the other excited states, so the $2 p \sigma_{u}$ state plays an important role in the ionization of the hydrogen molecular ions in the laser field. Interestingly, we find that the population of the excited states approaches zero as the laser pulse goes away, apart from the resonant cases. In the three-photon resonant case [Fig. 5(a)], the $1 s \sigma_{g}$ and $2 p \sigma_{u}$ states are strongly mixed when the laser field reaches its peak value and the population of the $2 p \sigma_{u}$ state does not go to zero as the laser field passes away. This is also true for the one-photon resonant peak, as shown in Fig. 5(c). It can be understood that, due to the nature of the resonance, the $1 s \sigma_{g}$ and $2 p \sigma_{u}$ states are strongly coupled together by the laser field and the final population of each state depends on how the laser field passes away. There is always noticeable population left in the $2 p \sigma_{u}$ state. This may provide another way to identify the ionization: the multiphoton resonant excitation process. It is different from the CREI peak in the time-dependent population of the $2 p \sigma_{u}$ state. Before the laser field reaches its peak, we can still identify the state as the $1 s \sigma_{g}$ state. When the laser field reaches its peak, the $1 s \sigma_{g}$ and $2 p \sigma_{u}$ states are mixed together by the laser field and we see that the electron shuttles between the two states and we cannot classify the states by the laser field-free state. How frequently the electron shuttles between the two states depends on the energy gap between 

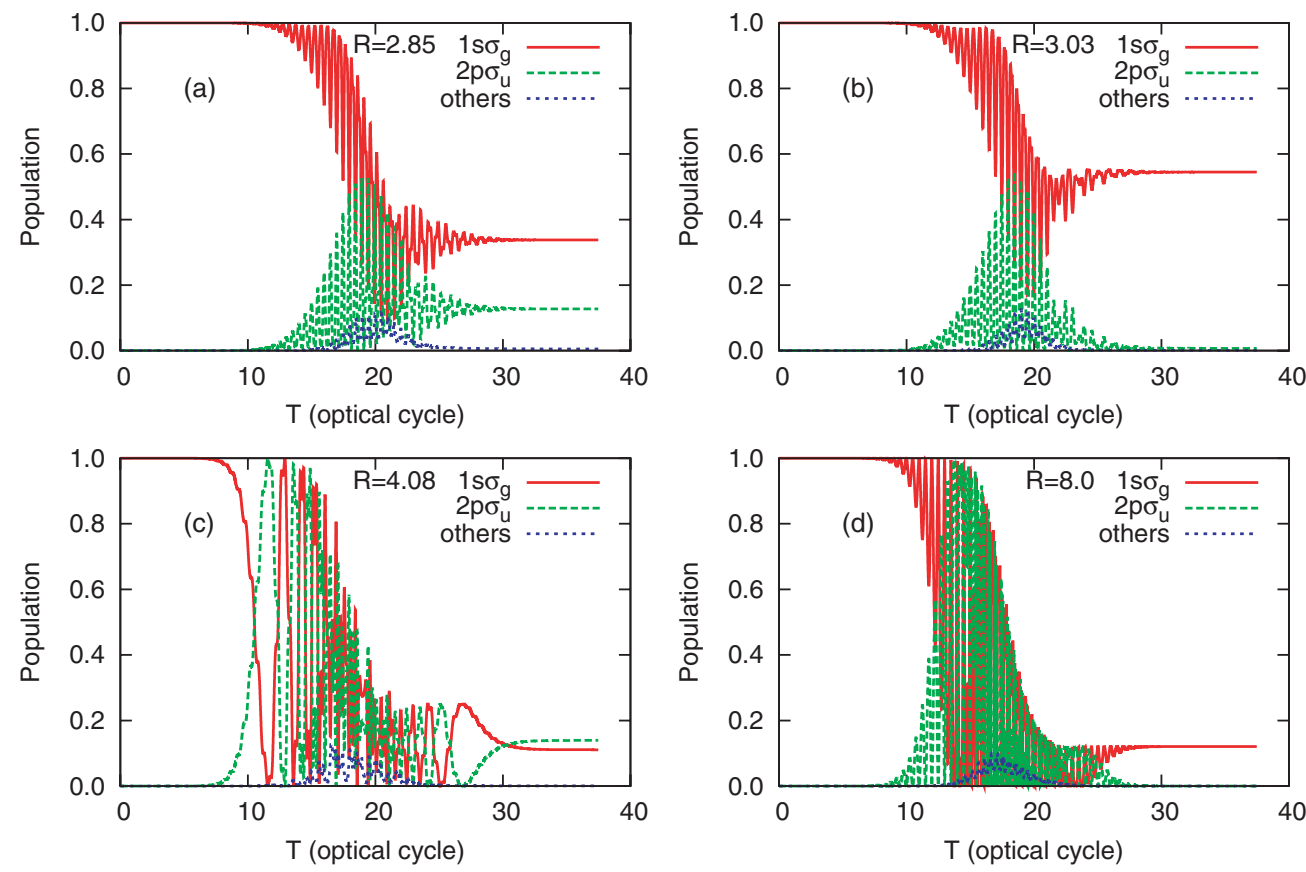

FIG. 5. (Color online) Time-dependent populations of the $1 s \sigma_{g}$ state (solid line), $2 p \sigma_{u}$ state (dashed line), and the sum of all the other electronic excited states (dotted line) at four different nuclear separations, which correspond respectively to (a) the three-photon resonant peak, (b) the minimum between the three-photon and one-photon resonant peaks, (c) the one-photon resonant peak, and (d) the CREI peak in Fig. 4.

them. The $2 p \sigma_{u}$ state plays a different role in each nuclear separation.

The vibrational wave packet, created through the ionization of hydrogen molecules by a short infrared laser pulse, was traced by a second infrared laser in the experiments of Refs. $[14,15]$. The complete motion of the vibrational wave packet can be recovered if an attosecond pulse is used as a probe light [23]. If we use two 10 -fs $480-\mathrm{nm}$ pulsed lasers with peak intensity of $3.5 \times 10^{14} \mathrm{~W} / \mathrm{cm}^{2}$ for the pump-probe experiment, can we get more information or better results? Figure 5 shows the simulated pump-probe KER spectra as a function of the time delay between the two lasers. Comparing with the pump-probe results using two 800-nm lasers (lower panel in Fig. 6), whenever the wave packet goes through the enhanced ionization region, the ionization is enhanced significantly. This property can be used to measure the vibrational period in the time domain and to obtain the vibrational wave packet information in the inner region. In this simulation, we assume that the initial wave packet follows the Franck-Condon distribution, since the laser field modifies the potential curve and the vibrational distribution may differ from the Franck-Condon distribution as shown in the experiment [24]. It is interesting to see how this affects the period of the wave packet in the experiment.

To summarize, we performed a theoretical study of the ionization of hydrogen molecular ions in a 480-nm pulsed laser field solving the time-dependent Schrödinger equation. Our simulated KER spectra are in agreement with the experimental measurements. Comparing the results with and without the $2 p \sigma_{u}$ state contribution, it was confirmed that the observed $10-\mathrm{eV}$ peak comes from the ionization via the three-photon resonant excitation to the $2 p \sigma_{u}$ state. Based on this mechanism, we suggested a pump-probe experiment to trace the vibrational wave packet.
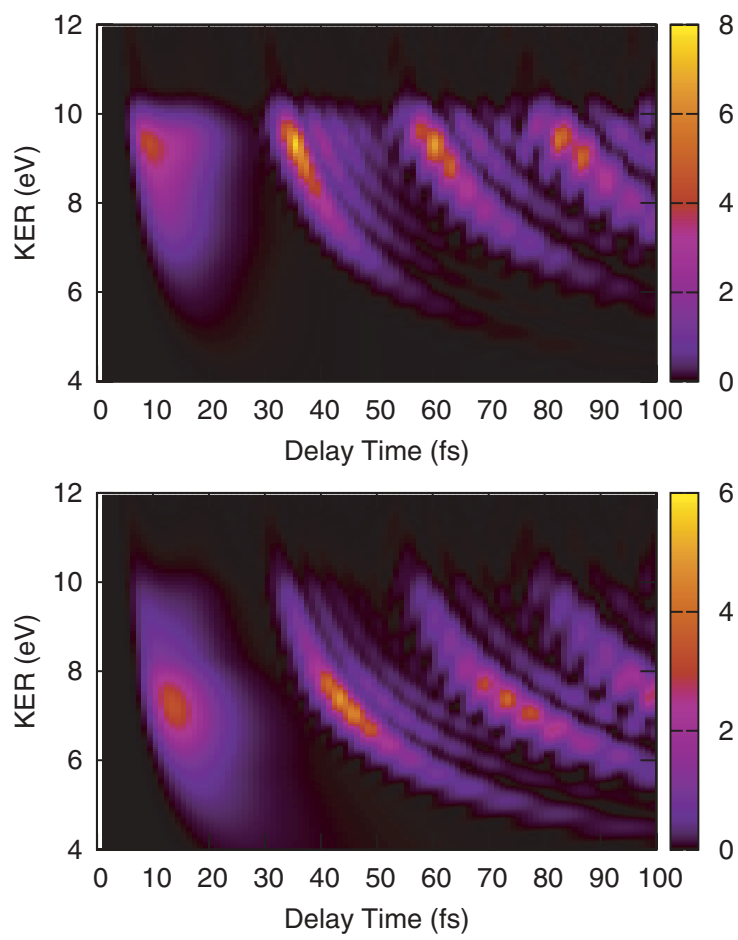

FIG. 6. (Color online) KER spectra as a function of the time delay between the pump and probe lasers. The lasers are 10-fs, 480- (upper panel) and $800-\mathrm{nm}$ (lower panel) pulsed lasers with a peak intensity of $3.5 \times 10^{14} \mathrm{~W} / \mathrm{cm}^{2}$. 


\section{ACKNOWLEDGMENTS}

This research was supported by a Grand-in-Aid for Scientific Research (C) from the Japan Society for the Promotion of Science. The numerical calculations were carried out by the T2K-Tsukuba System in the Center for Computational Sciences, University of Tsukuba, Japan. We thank I. Litvinyuk for providing the experimental data.
[1] H. Niikura, F. Legare, R. Hasbani, A. D. Bandrauk, M. Y. Ivanov, D. M. Villeneuve, and P. B. Corkum, Nature 417, 917 (2002).

[2] A. S. Alnaser, T. Osipov, E. P. Benis, A. Wech, B. Shan, C. L. Cocke, X. M. Tong, and C. D. Lin, Phys. Rev. Lett. 91, 163002 (2003).

[3] X. M. Tong, Z. X. Zhao, and C. D. Lin, Phys. Rev. Lett. 91, 233203 (2003).

[4] A. S. Alnaser, X. M. Tong, T. Osipov, S. Voss, C. M. Maharjan, P. Ranitovic, B. Ulrich, B. Shan, Z. Chang, C. D. Lin et al., Phys. Rev. Lett. 93, 183202 (2004).

[5] P. B. Corkum, Phys. Rev. Lett. 71, 1994 (1993).

[6] H. Niikura, F. Legare, R. Hasbani, M. Y. Ivanov, D. M. Villeneuve, and P. B. Corkum, Nature 421, 826 (2003).

[7] T. Zuo and A. D. Bandrauk, Phys. Rev. A 52, R2511 (1995).

[8] G. N. Gibson, M. Li, C. Guo, and J. Neira, Phys. Rev. Lett. 79, 2022 (1997).

[9] F. Legare, I. V. Litvinyuk, P. W. Dooley, F. Quere, A. D. Bandrauk, D. M. Villeneuve, and P. B. Corkum, Phys. Rev. Lett. 91, 093002 (2003).

[10] X. M. Tong and C. D. Lin, Phys. Rev. A 70, 023406 (2004).

[11] I. V. Litvinyuk, A. S. Alnaser, D. Comtois, D. Ray, A. T. Hasan, J.-C. Kieffer, and D. M. Villeneuve, New J. Phys. 10, 083011 (2008).
[12] X. M. Tong and S. I. Chu, Chem. Phys. 217, 119 (1997).

[13] X. Chu and Shih-I Chu, Phys. Rev. A 64, 063404 (2001).

[14] A. S. Alnaser, B. Ulrich, X. M. Tong, I. V. Litvinyuk, C. M. Maharjan, P. Ranitovic, T. Osipov, R. Ali, S. Ghimire, Z. Chang, C. D. Lin, and C. L. Cocke, Phys. Rev. A 72, 030702(R) (2005).

[15] T. Ergler, A. Rudenko, B. Feuerstein, K. Zrost, C. D. Schroter, R. Moshammer, and J. Ullrich, Phys. Rev. Lett. 95, 093001 (2005).

[16] J. Wang, Shih-I Chu, and C. Laughlin, Phys. Rev. A 50, 3208 (1994).

[17] D. A. Telnov and Shih-I Chu, Phys. Rev. A 59, 2864 (1999).

[18] X. M. Tong and C. D. Lin, Phys. Rev. Lett. 98, 123002 (2007).

[19] I. Kawata, H. Kono, and Y. Fujimura, J. Chem. Phys. 110, 11152 (1999).

[20] X. Chu and Shih-I Chu, Phys. Rev. A 63, 013414 (2000).

[21] X. M. Tong, Z. X. Zhao, and C. D. Lin, Phys. Rev. A 66, 033402 (2002).

[22] S. Augst, D. D. Meyerhofer, D. Strickland, and S. L. Chin, J. Opt. Soc. Am. B 8, 858 (1991).

[23] X. M. Tong and C. D. Lin, Phys. Rev. A 73, 042716 (2006).

[24] X. Urbain, B. Fabre, V. M. Andrianarijaona, J. Jureta, J. H. Posthumus, A. Saenz, E. Baldit, and C. Cornaggia, Phys. Rev. Lett. 92, 163004 (2004). 